\title{
Building an Ethics in Computing Website Using Peer Review
}

\author{
Edward F. Gehringer \\ North Carolina State University \\ efg@ncsu.edu
}

Abstract

An Ethics in Computing Website covering almost 100 topics has been developed using peerreviewed student contributions. Students in the author's one-credit Ethics in Computing course select a topic to research from a list provided by the instructor, or propose a topic of their own choosing. Their contributions are then reviewed, and ultimately graded, by three other students taking the course. The best-reviewed pages are then incorporated into the Website. However, most of the work of maintaining the site is performed by a set of independent-study projects during the 10-week summer session. Each student chooses a set of topics, and completes one topic every two weeks. Each submission is subjected to two rounds of review, one round per week. Some of the topics are new; others are merely updates to existing pages. The amount of work required on each topic is ranked from 1 to 5 , with 5 being the highest. Each student is expected to complete topics with a set total rank, usually about 15 . In addition to researching topics, each student chooses one "special job," such as improving the graphics, installing a search engine, or developing a set of style guidelines. The project can be supervised by graduate students, who undertake the responsibility of assigning work to students and integrating the work into the site. Benefits of the project include (i) giving the students an in-depth look at several different ethical issues, (ii) constructing a resource that has been used by instructors around the world, and (iii) providing a low-overhead mechanism for adding another course to the curriculum. This methodology should be applicable to courses involving professional issues in all areas of engineering.

\section{Introduction}

Ethics in Computing is a fast-changing field. The "hot issues" of one year frequently were not even on the radar screen the year before. The past seven years have seen the rise and fall of encryption policy (e.g., the Clipper chip), regulation of Internet content (e.g., the Communication Decency Act), and Y $2 \mathrm{~K}$ as issues of the hour, that an up-to-date course in computer ethics seemed obligated to address. Concurrently, more enduring issues like spam, copyright of electronic materials, and the "digital divide" have risen to prominence. Clearly, it is difficult for textbooks to keep up. Texts can provide the basic principles and ethical theory, but to hold the attention of a class, it is important to supplement them with current readings.

Since the mid-'90s, a plethora of relevant articles have been available on the Web. These are readily available with a few clicks on any search engine. But, search engines alone do not fill the bill. The instructor is unlikely to know the right search terms to uncover some of the best case studies, like the Hughes Aircraft case [Bowy 00], the best illustrative sites, like Tom Darby's interactive tour of the Internet worm [Darb 95], or ethical issues related to fields like artificial intelligence or e-commerce. Moreover, it is easy to fail to notice new ethical issues as they arise 
with advances in technology (how many people recognized MP3s as an ethical issue two years ago?).

These factors suggest that it would be valuable to have a Website that contains links to articles on various topics and analyses of these topics. New pages can be developed as new topics arise, and the best links on each topic can be preserved as long as they are relevant. This paper is about the construction of such a site, which began in 1996 and has been periodically updated.

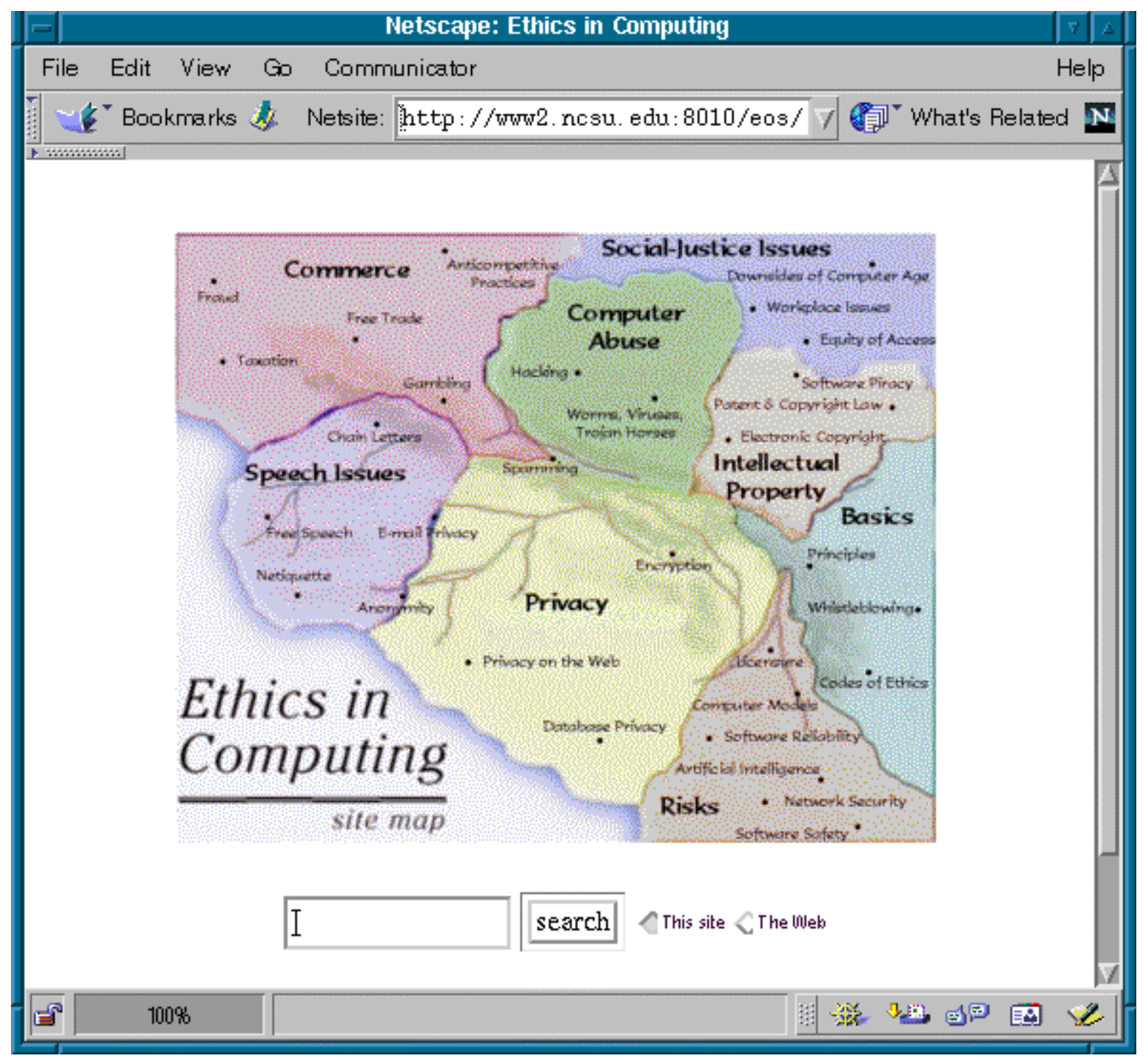

Figure 1. Homepage of site

\section{The site}

Our Ethics in Computing Website, http://www2 ncsu.edu/eos/info/computer_ethics (homepage, Figure 1) is divided into eight major areas: Basics, intellectual property, computer abuse, speech issues, privacy, risks, commerce, and social-justice issues. These cover the same 
topics as the ACM Computing Curricula 2001 requirements for SP: Social, Ethical and Professional Issues, though the grouping is slightly different. Each area is subdivided into various topics (Figure 2). Each of the covered topics has a "table of contents" page (Figure 3), containing links to relevant articles, and a study guide (Figure 4), which attempts to identify some of the major issues. Many of the topics also have discussion questions (Figure 5).

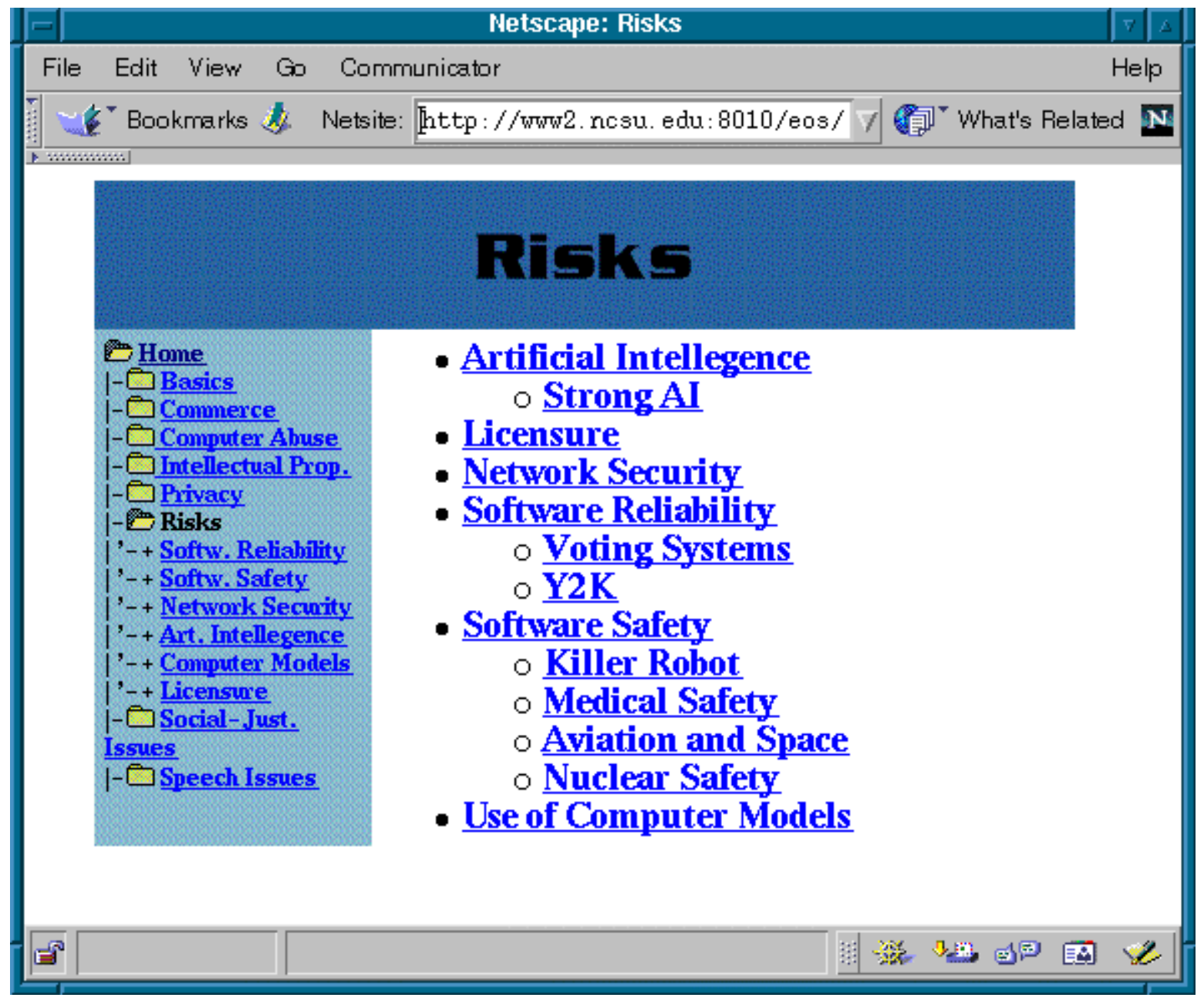

Figure 2. Topics in Risks area

\section{The methodology}

At the end of each semester, each student in the author's Ethics in Computing class does a project involving researching a topic in computer ethics and building Table of Contents and study-guide pages. The students select from a list of topics provided by the instructor and/or the teaching assistant. To assure that all the topics are covered, only two or three students are allowed to sign up for any particular topic. (Students are also allowed to select a topic of their own choosing that is not on the list, but never have more than one or two students done this.) The projects are peer-reviewed, using the author's Web-based PG application [Gehr 99, Gehr 


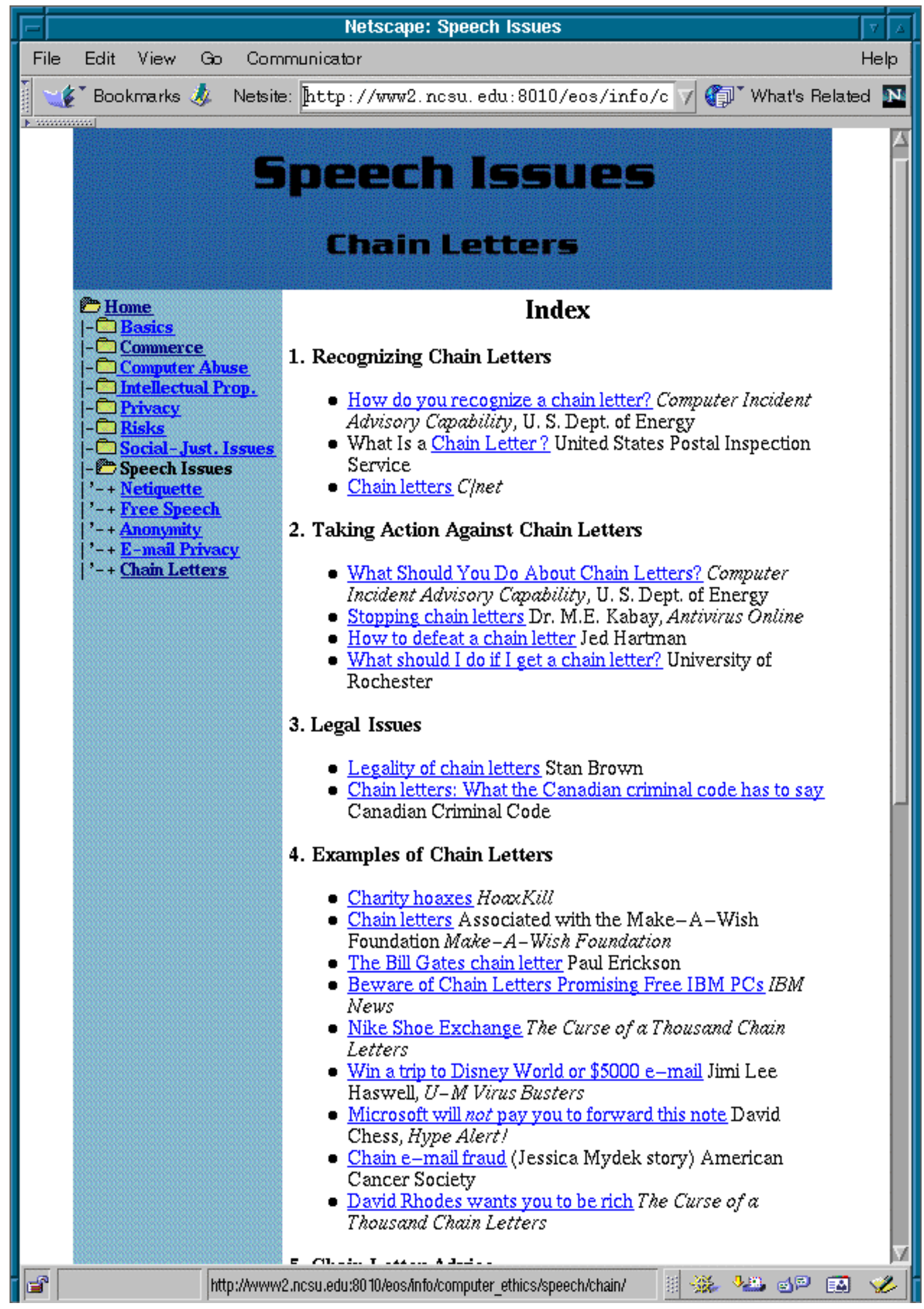

Figure 3. A "Table of Contents" page

Proceedings of the 2001 American Society for Engineering Education Annual Conference \& Exposition Copyright (C) 2001, American Society for Engineering Education 


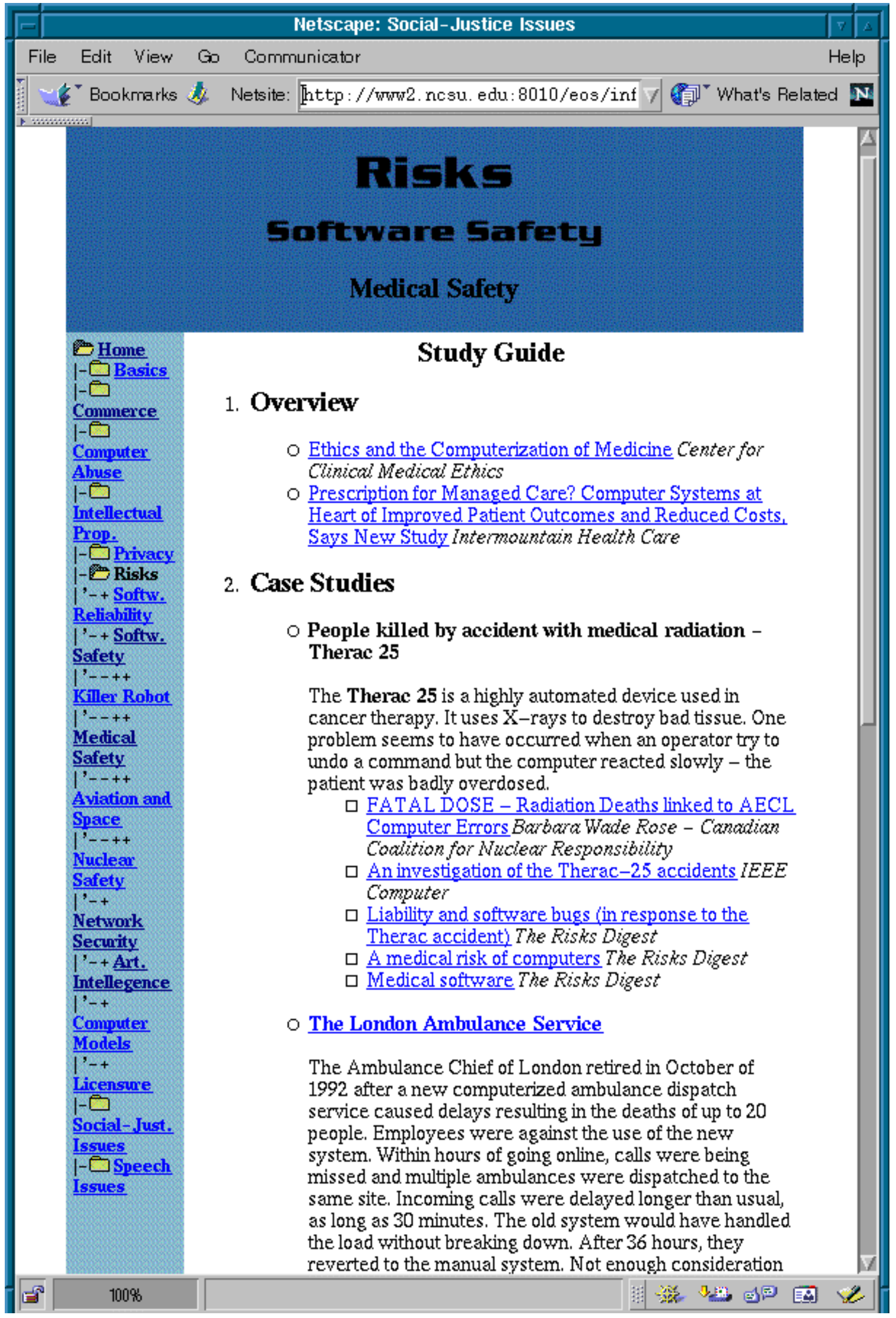

Figure 4. A study guide

Proceedings of the 2001 American Society for Engineering Education Annual Conference \& Exposition Copyright (C) 2001, American Society for Engineering Education 


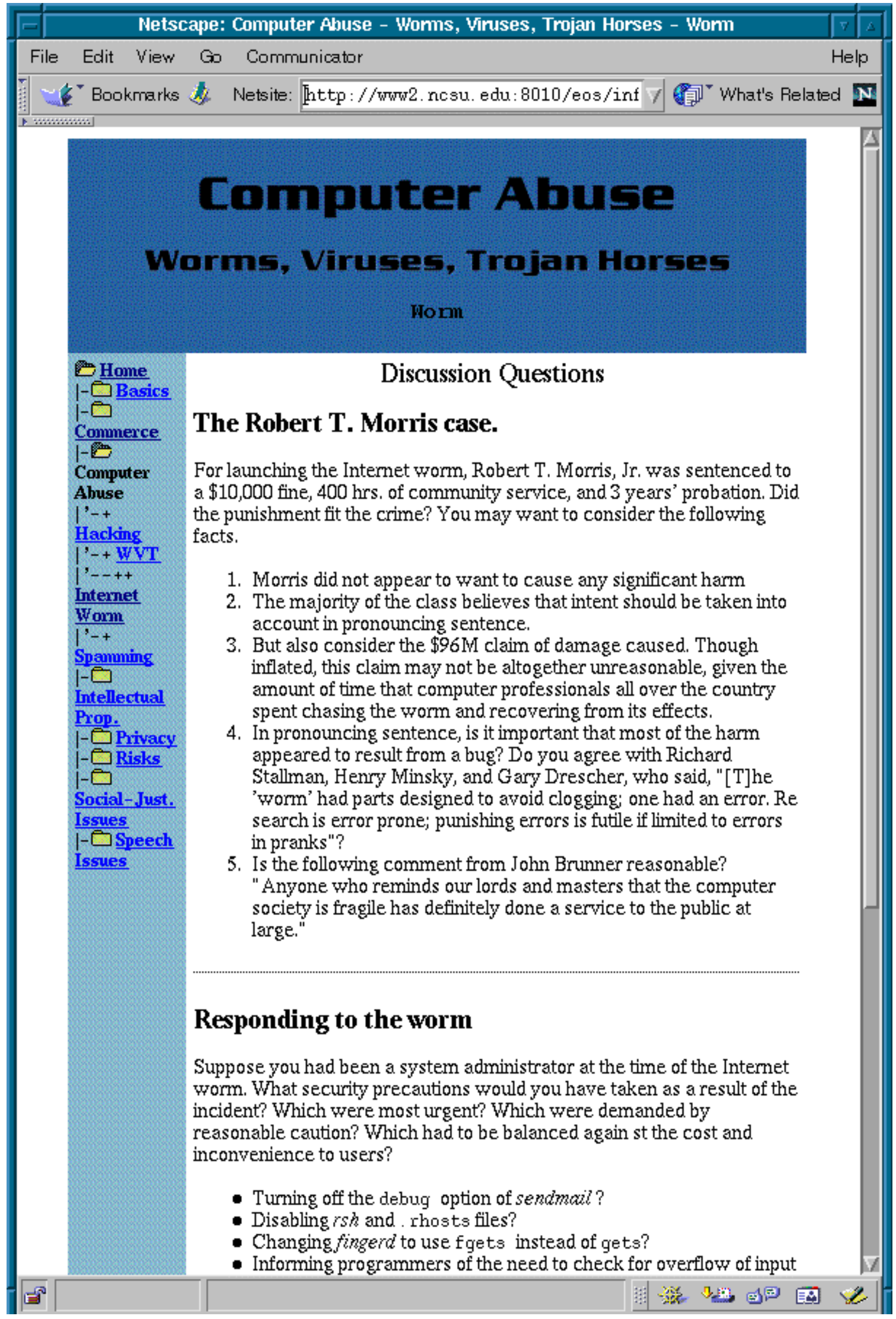

Figure 5. Discussion questions

Proceedings of the 2001 American Society for Engineering Education Annual Conference \& Exposition Copyright (C) 2001, American Society for Engineering Education 
00]. Typically, each student is assigned to review three projects, one or two of which are on the same topic (s)he has chosen. Assuming everyone does the assigned reviews, this assures that each project is reviewed both by "experts" who have also researched the topic and "members of the general public," who haven't. At the end of the review period, the reviewers assign grades to their authors. If the reviewers are in general agreement about the quality of the project, their grades are used; if not, the instructor takes a peek and makes a final decision. In addition to giving students the experience of writing for their peers, peer review and peer grading is the only way to get the work done in such a large class, whose enrollment has ranged from 90 to 120 students in recent semesters.

Although the class projects provide a lot of raw material for the Website, most of the real work has been done in a series of summer projects, involving from four to ten students per year. Currently, this is structured as a special section of an individual-topics course (CSC 495) during the ten-week summer session. Last summer, two of the students were graduate students, receiving master's-level credit for serving in a supervisory role.

In the first two weeks of the summer session, each undergraduate is responsible for merging the student projects in one of the eight major areas. This involves creating a page of pointers to each topic in the area, appropriately structured using the site's style guidelines. The student is allowed to use the highest-ranked submission on each topic, but is encouraged to construct a hybrid page for each topic, including the best links and analyses from each of the student projects on that topic. A short README file is created to describe how the projects were merged.

Concurrently, the graduate students are assigned to go through the existing Website, as well as the new student projects, and decide how much further work is required to bring each topic up to date. Topics can require a lot of work if the existing pages are badly done, or if a lot of new developments have occurred during the year. Each topic is assigned a score from 1 to 5, depending on how much work is required (with " 5 " denoting the most work). In recent years, speech issues like spam and Internet filters have tended to change rapidly, while other topics like codes of ethics and artificial intelligence have tended to remain fairly static.

After the second week, the undergraduate students choose a set of topics (usually four topics but occasionally more) whose scores add up to a certain quota (generally 14 to 16). Last summer, each undergraduate was told to choose one topic each from those scored 2, 3, 4, and 5, with the graduate students doing quick updates on all the topics scored 1. The remaining eight weeks are divided into four rounds of two weeks each. In each round, each undergraduate is responsible for completing one topic. In the first week of each round, students are required to submit their topic for review. During the second week, reviewers and authors communicate via a shared Web page, with the reviewers suggesting improvements that the authors can make. At the end of the second week, the reviewers assign each of their authors a grade. The graduate students' work on the topics scored 1 was not subjected to peer review last summer; this was a mistake, as it would have helped in assuring that it was completed.

In addition to the weekly submissions of Web pages, each student is responsible for one "special job" during the term. These vary from year to year. Examples from past years have included-

- Installing a search engine. 
- Installing a program to find dead links.

- Redesigning the Web page, improving the graphics and the navigation aids.

- Maintaining style guidelines for the Website.

- Searching the Web for names and e-mail addresses of other ethics-in-computing instructors to whom we can advertise our Website.

- Taking ethics-in-computing textbooks and building index of which chapters are covered under which topics on the Website.

- Integrating the new pages into the Website. It turns out that this is too large a job for one person to do, since it involves quality control on the whole Website. In the future, the person chosen to perform this function will probably not be required to research weekly topics as well.

Weekly meetings are held throughout the summer. Attendance is expected, but not strictly required, since many of the students also have summer jobs. In fact, one of the advantages of a project like this is that it can be carried out anywhere where Web access is available; one summer the student who did best on the project was on co-op in Colorado. The meetings are an hour long, and are usually held late in the afternoon. They are conducted in a classroom with Internet access and a projector so that the submissions can be visited during the meeting. During the meetings, the instructor reviews pages at random, making comments on style and substance. We discuss problems that have arisen, and students give tips to each other. We get reports on the progress on the special jobs.

Grades in this class are based on-

- $\quad$ peer grades of the pages submitted by the student,

- the percentage of assigned reviews that the student has done, and peer grades assigned to these reviews by other students.

- the quality of work on the "special job" (which is graded by the instructor), and

- attendance at weekly meetings.

Not only does this class help us maintain the Website, and thereby provide a service to the computer-ethics community, it also allows our students to get more in-depth experience analyzing ethical issues than they get in their required classes. Finally, it helps meet a need for additional summer classes that are convenient for students who have summer jobs. It does all of these with minimal demands on the instructor's time (probably two to three hours/wk.).

\section{Challenges and problems}

As we get more experience with this methodology, we attempt to refine it to deal with problems that arise. Early on, it was discovered that there needed to be due dates for each individual topic; otherwise, nothing would be completed until the last couple of weeks, and many topics would not be completed at all.

It is difficult to predict how well students will do on a project like this. Their performance does 
not correlate well with their performance on more technical courses. The best students are frequently those with minors in the humanities; however, there are not many of these. Students tend to do a better job of researching ethical issues that are presented to them than at finding new ethical issues. For example, I have seen several Web pages on e-commerce, artificial intelligence, and nuclear safety that contain links to many good articles describing the topics, but none identifying any ethical issues. The nuclear-safety topic brings up another difficulty: the intent was to study the role of software in maintaining safety, but most of the links the students found related to general safety issues in nuclear power-and even nuclear weapons. Even when reminded that the Website focuses on ethics in computing, the students evidently include links because they are interesting, rather than because they are topical.

Part of the problem may be that some topics are just difficult to research on the Web. One example seems to be anticompetitive uses of databases. We have not been able to find more than one or two articles on unfair use of computerized airline reservation systems or medical-supply databases like Baxter International's [Spin 95]. Expert systems is another example; it is nearly impossible to find Web references to the ethics of allowing software to make life-or-death decisions on treating patients, despite the fact that related print articles are not difficult to find. The same situation used to prevail regarding licensure of software engineers, but fortunately this is changing. For some of these topics, we have scanned in print articles for use in our own classes, but copyright restrictions, of course, prohibit us from putting them on the Web.

On controversial topics where Web references abound, there is still the problem of choosing appropriate ones. Our guidelines state that all major points of view should be represented, but if one viewpoint is much more common on the Web than another, our Website should not attempt to hide that fact. Naturally, though, students tend to overrepresent their own viewpoint. So on the issue of pornography, we have gone from having a page emphasizing the dangers of restricting speech, to one stressing the dangers of pornography, and back again, depending on who did the most recent revision. There have been several cases where the only "con" article on a particular issue has been one setting up "strawmen" that can be refuted later on in the article. Thus, the site administrator always needs to pay particular attention to areas of controversy.

We have not been able to maintain very uniform quality in the study guides. Evidently the problem is that reviewers tend to concentrate on the table of contents page on a particular topic, and if this is good, they give a high score regardless of how much attention has been devoted to the study guide. The obvious solution is to give a separate grade for the study guide; this will be implemented in the next offering of the course.

Uniformity of style is also difficult to maintain. This is a particular problem in the citation of sources. The guidelines say that for each link, both the site and the author (if known) should be identified. Unfortunately, there are many cases where neither is cited, or where a university is cited as the source, instead of the faculty member(s) who wrote the article.

Integration of the site turned out to be more difficult than we expected. Students who are updating a page may not realize the importance of certain links on that page, and thus they remove them. Or, a Website may be temporarily down, causing the student to assume that the link is broken. Whatever the explanation, some of the best links are often removed by students updating a page. Thus, when integrating the pages, it is necessary to go back to the original page 
as well as all of the new submissions, and merge them, using editorial discretion to prevent the page from becoming too long. This makes integration a big job, and one that takes a long time. It usually drags on into the next semester. This, in turn, keeps some pages from making it to the site until they are several months old, by which time some of the links are broken. In the future, therefore, one of the best students will be assigned the sole task of integrating Web pages into the site.

\section{Conclusion}

We have described a Website on ethics in computing and the methodology for creating it. It provides a repository for articles and analyses of dozens of topics related to that field. The methodology should be usable for creating a Website on professional or public-policy issues in any field. The fact that it is created by peer review allows it to be constructed with a minimum of faculty time. The management of such a site, however, raises a number of issues that must be attended to. We have delineated some of them in the paper. We believe that these techniques provide an effective way to create educational resources that can improve the quality of courses addressing the non-technical aspects of curricula in all fields of engineering.

\section{Bibliography}

[Bowy 00] Bowyer, Kevin W., "Goodearl and Aldred versus Hughes Aircraft: A whistle-blowing case sudy," Proc. Frontiers in Education 2000, Session S2F.

[Darb 95] Thomas Darby and Charles Schmidt, “The Morris Internet Worm," currently accessible at http://www2 .ncsu.edu/eos/info/computer_ethics/abuse/wvt/worm/darby.

[Gehr 99] Gehringer, Edward F., "Peer grading over the Web: enhancing education in design courses," Proc. ASEE 1999, Session 2532.

[Gehr 00] Gehringer, Edward F., "Strategies and mechanisms for electronic peer review," Proc. Frontiers in Education 2000, Session F1B.

[Spin 95] Spinello, Richard A., Ethical Aspects of Information Technology, Prentice-Hall, 1995.

\section{EDWARD F. GEHRINGER}

Edward Gehringer is an associate professor in the Department of Electrical and Computer Engineering and the Department of Computer Science at North Carolina State University. He has been a frequent presenter at educationbased workshops in the areas of computer architecture and object-oriented systems. His research interests include architectural support for persistence and large object systems, memory management and memory-management visualization, and garbage collection. He received a B.S. from the University of Detroit(-Mercy) in 1972, a B.A. from Wayne State University, also in 1972, and the Ph.D. from Purdue University in 1979. 